\title{
Kohlenhydratrestriktion und Wachstumsstörung bei zwei Kindern mit Diabetes mellitus Typ 1: ein Fallbericht
}

\author{
Roberto Franceschi ${ }^{a}$ Caterina Rizzardi ${ }^{a}$ Vittoria Cauvin ${ }^{a}$ Federica Berchielli ${ }^{b}$ \\ Alice Liguori $^{\mathrm{a}}$ Massimo Soffiati $^{\mathrm{a}}$ \\ a Pediatric Diabetology Unit, S. Chiara Hospital of Trento, Trento, Italien; \\ ${ }^{b}$ Nutrition and Dietetics Unit, S. Chiara Hospital of Trento, Trento, Italien
}

\section{Schlüsselwörter}

Kohlenhydratrestriktion · Wachstumsstörung · Typ-1-Diabetes

\begin{abstract}
Zusammenfassung
Zahlreiche Medien haben restriktive Diäten bei Diabetes mellitus populär gemacht und berichtet, dass Kohlenhydrate die Ursache für Blutzuckerspitzen, extrem hohe Insulin-Dosierungen und Gewichtszunahme sind. In der vorliegenden Arbeit berichten wir über zwei Fälle von Kindern mit Diabetes mellitus Typ 1, bei denen die Einhaltung einer streng kohlenhydratreduzierten Diät Wachstumsstörungen zur Folge hatte. Zwei präpubertäre Patienten mit Typ1-Diabetes zeigten bei der Kontrolluntersuchung während der Honeymoon-Phase eine fehlende Gewichtszunahme und geringe Wachstumsgeschwindigkeit. Die Ernährungsanalyse ergab, dass eine kohlenhydratarme Diät begonnen worden war, um eine Reduzierung der postprandialen Blutzuckerspitzen zu erreichen.
\end{abstract}

Nach Zufuhr entsprechender Kohlenhydratmengen begannen das Gewicht und die Körpergröße der beiden Patienten zu steigen. Es liegen nur wenige veröffentlichte Daten vor, die dafür sprechen, die Kohlenhydratzufuhr nicht zu begrenzen; darum berichten wir in der vorliegenden Arbeit über zwei Fälle von Kindern mit Typ-1-Diabetes, bei denen die Einhaltung einer solchen Ernährungsform zu Wachstumsstörungen geführt hat. Besonders hervorheben möchten wir, dass dabei die Schulung der Familien von Kindern mit Typ1-Diabetes zu diesem Aspekt eine wichtige Rolle spielt, vor allem wenn sich die Kinder gerade in der Honeymoon-Phase befinden oder diese zu Ende geht.

(c) 2020 The Author(s). Published by S. Karger AG, Basel

\section{Einführung}

Zahlreiche Medien haben restriktive Diäten bei Diabetes mellitus Typ 1 und 2 populär gemacht und berichtet, dass Kohlenhydrate die Ursache für Blutzuckerspitzen, extrem hohe Insulin-Dosierungen und Gewichtszunahme sind. In einer kürzlich veröffentlichten Arbeit wurde der Fall eines Kindes mit Typ-1-Diabetes beschrieben, das erfolgreich mit einer stark kohlenhydratreduzierten Diät behandelt wurde, wodurch es zu einer Normalisierung der HbAlc-Werte und optimalen Time-in-Range (TIR, Zeit im Zielbereich)-Werten kam [1]. Dagegen kann eine kohlenhydratreduzierte Diät den internationalen Leitlinien zufolge bei pädiatrischen Patienten mit Typ-1-Diabetes aufgrund der geringen Energieaufnahme Wachstumsstörungen, ein höheres Lipidrisikoprofil, psychische Komorbiditäten und soziale Isolation zur Folge haben $[2,3]$. Um postprandialen Hyperglykämien entgegenzuwirken, empfehlen die Leitlinien zudem, die Einhaltung einer ausgewogenen Ernährung zu fördern, Kohlenhydrate mit niedrigem glykämischen Index (GI) durch solche mit hohem GI zu ersetzen und die Aufnahme von Ballaststoffen zu steigern. Au- 
ßerdem sollte darauf geachtet werden, dass die präprandiale Bolus-Gabe nicht zu spät erfolgt, ein optimales Insulin-Kohlenhydrat-Verhältnis besteht und dass die Zahl der Zwischenmahlzeiten reduziert wird [2,3]. Da nur wenige veröffentlichte Daten vorliegen, die dafür sprechen, die Kohlenhydratzufuhr nicht zu begrenzen, berichten wir in der vorliegenden Arbeit über zwei Fälle von Kindern mit Typ-1-Diabetes, bei denen die Einhaltung dieser Diät Wachstumsstörungen zur Folge hatte.

\section{Fallbericht}

Bei Patient 1 wurde im Alter von 5 Jahren und 4 Monaten im pädiatrischen Diabeteszentrum in Trento (Italien) ein Typ-1-Diabetes diagnostiziert. Es fanden sich eine Hyperglykämie (622 mg/ dl), Polyurie, Polydipsie, Polyphagie, Azidose (pH-Wert: 7,25) sowie ein Gewichtsverlust von 1,2 kg über einen Zeitraum von 4 Wochen. Der HbA1c-Wert lag zum Zeitpunkt der Diagnosestellung bei 9,2\% (77 mmol/mol), das Körpergewicht betrug 18,3 kg (-0,88 SDS), seine Körpergröße $114 \mathrm{~cm}$ (0,04 SDS) und sein Body Mass Index (BMI) $14 \mathrm{~kg} / \mathrm{m}^{2}$ (1,1 SDS). Der C-Peptid-Spiegel lag bei $0,1 \mathrm{nmol} / 1$ und die Untersuchung auf Autoantikörper für Typ1-Diabetes fiel positiv aus. Es wurde eine Behandlung mit mehrmaligen täglichen Injektionen unter Verwendung des Kohlenhydrat-Insulin-Verhältnisses zu den Mahlzeiten und morgendlicher Gabe von Glargin begonnen, und der tägliche Gesamtinsulinbedarf lag bei $0,7 \mathrm{U} / \mathrm{kg}$. Zwei Monate nach der Diagnosestellung betrug der HbAlc-Wert $50 \mathrm{mmol} / \mathrm{mol}$ und der tägliche Gesamtinsulinbedarf 0,3 U/kg, das heißt der Patient befand sich definitionsgemäß in der Honeymoon-Phase (Zeitraum mit einem Insulinbedarf von weniger als $0,5 \mathrm{U} / \mathrm{kg} / \mathrm{Tag}$ und einem HbAlc-Wert von weniger oder gleich $53 \mathrm{mmol} / \mathrm{mol}$ ). Die Behandlung wurde auf eine Insulinpumpentherapie umgestellt, weil er zu den verschiedenen Tageszeiten unterschiedliche Basalraten benötigte. Die Mutter war im Umgang mit der Pumpe und in Bezug auf das Ernährungswissen kompetent.

Bei der Kontrolluntersuchung im Alter von 6 Jahren und 2 Monaten (nach einer Diabetes-Krankheitsdauer von 10 Monaten) zeigte er keine Gewichtszunahme (18,3 kg; -1,26 SDS) und das Längenwachstum war zum Stillstand gekommen (114 cm; -0,52 SDS). Die Schilddrüsenuntersuchungen fielen normal aus und das Screening auf Zöliakie war negativ. Ein 24-h-Recall (24-h-Erinnerungsprotokoll) ergab eine gute Energieaufnahme und ein breites Spektrum an Nahrungsmitteln. Die ausgelesenen Insulinpumpendaten zeigten einen sehr geringen Bolus zu den 3 Hauptmahlzeiten, was jedoch zur Honeymoon-Phase passen würde. Die Gesamtinsulindosis betrug 0,27 U/kg/Tag und der HbAlcWert $50 \mathrm{mmol} / \mathrm{mol}$.

Drei Monate später (nach einer Diabetes-Krankheitsdauer von 13 Monaten) hatte sein Gewicht nicht signifikant zugenommen (19,3 kg; -1,23 SDS) und die Körpergröße war gleich geblieben $(114 \mathrm{~cm}$; -1,06 SDS); der HbAlc-Wert betrug $45 \mathrm{mmol} / \mathrm{mol}$, die Time-inRange (TIR) 72\%, die Zeit in Hypoglykämie 4\% und die Gesamtinsulindosis lag bei $0,35 \mathrm{U} / \mathrm{kg} / \mathrm{Tag}$. Es wurden eine Magnetresonanztomographie des Gehirns und der Hypophyse sowie eine
Untersuchung der Wachstumshormone durchgeführt, die ebenso normal ausfielen wie die Schilddrüsentests, die Bestimmung des Cortisolspiegels und das Zöliakie-Screening. Die Lipidwerte lagen mit einem Gesamtcholesterin von $151 \mathrm{mg} / \mathrm{dl}$, einem HDLWert von $52 \mathrm{mg} / \mathrm{dl}$ und Triglyceridwerten (TGD) von $65 \mathrm{mg} / \mathrm{dl} \mathrm{im}$ Normbereich.

Schließlich wurde er auf die Kinderstation aufgenommen, um die Energiezufuhr zu überprüfen. Erst zu diesem Zeitpunkt gab die Mutter an, dass das Kind eine kohlenhydratarme Kost (30 g/Tag) erhielt, um die Zahl der postprandialen Blutzuckerspitzen in der Honeymoon-Phase zu verringern und die Funktion der Betazellen zu erhalten. Die Ernährungsanalyse anhand eines 3-tägigen Ernährungsprotokolls ergab, dass die Gesamtenergieaufnahme bei etwa $70 \%$ des geschätzten Energiebedarfs von $1500 \mathrm{kcal}$ lag. Dabei stammten 12\% der gewonnenen Energie aus Kohlenhydraten, $27 \%$ aus Protein und $61 \%$ aus Fett.

Das Diabetesteam klärte die Mutter über die Bedeutung einer gesunden Ernährung für Wachstum, Lipidprofil und psychologische Aspekte auf. Bei den folgenden Kontrollterminen wurde eine liberalere Kohlenhydratzufuhr von 190 g/Tag angegeben und das Wachstum nahm ab dem Alter von 6 Jahren und 10 Monaten (nach einer Diabetes-Krankheitsdauer von 18 Monaten) zu, wie in Abbildung 1 dargestellt. Der Patient hatte für etwa 10 bis 12 Monate eine kohlenhydratarme Kost erhalten. Beim letzten Kontrolltermin hatte Patient 1 ein Gewicht von $29 \mathrm{~kg}$ (-0,39 SDS), eine Größe von $134 \mathrm{~cm}$ (0,03 SDS), einen BMI von $16,15 \mathrm{~kg} / \mathrm{m}^{2}(-0,54$ $\mathrm{SDS}$ ) sowie einen HbAlc-Wert von $50 \mathrm{mmol} / \mathrm{l}$ und die Gesamtinsulindosis lag bei $0,68 \mathrm{U} / \mathrm{kg} / \mathrm{Tag}$.

Bei Patientin 2 wurde im Alter von 6 Jahren und 6 Monaten im pädiatrischen Diabeteszentrum in Trento (Italien) ein Typ-1-Diabetes diagnostiziert. Es fanden sich eine Hyperglykämie (476 mg/dl), Polyurie, Polydipsie, Azidose (pH-Wert: 7,25) sowie ein Gewichtsverlust von $2 \mathrm{~kg}$ über einen Zeitraum von 3 Wochen. Der HbAlc-Wert lag zum Zeitpunkt der Diagnosestellung bei 14,2\% (132 mmol/mol), das Körpergewicht betrug 26,5 kg (0,67 SDS), ihre Körpergröße $130 \mathrm{~cm}$ (1,93 SDS) und der BMI 15,7 kg/m². Der C-Peptid-Spiegel lag bei $0,05 \mathrm{nmol} / \mathrm{l}$ und die Untersuchung auf Autoantikörper für Typ-1-Diabetes fiel positiv aus. Es wurde eine Behandlung mit mehrmaligen täglichen Injektionen unter Verwendung des Kohlenhydrat-Insulin-Verhältnisses zu den Mahlzeiten und morgendlicher Gabe von Glargin begonnen, und der tägliche Gesamtinsulinbedarf lag bei 0,8 U/kg.

Bei den Kontrolluntersuchungen in den folgenden Monaten waren ein Gewichtsverlust sowie eine geringe Wachstumsgeschwindigkeit zu beobachten; Durchfall oder Erbrechen wurden nicht angegeben. Die Honeymoon-Phase begann 3 bis 4 Monate nach der Diagnose (0,42 U/kg/Tag). Im Alter von 8 Jahren (nach einer Diabetes-Krankheitsdauer von 18 Monaten) betrug ihr Körpergewicht 22,9 $\mathrm{kg}(-1,08$ SDS), die Körpergröße $133 \mathrm{~cm}$ (0,89 SDS) und der BMI 12,9 kg/m² (1,68 SDS). Die Schilddrüsentests und die Bestimmung der Cortisolspiegel waren normal und das Screening auf Zöliakie fiel negativ aus. Die Lipidwerte lagen mit einem Gesamtcholesterinwert von 158 mg/dl, einem HDL-Wert von 58 $\mathrm{mg} / \mathrm{dl}$ und einem Trigylzeridwert von $58 \mathrm{mg} / \mathrm{dl}$ im Normbereich. 


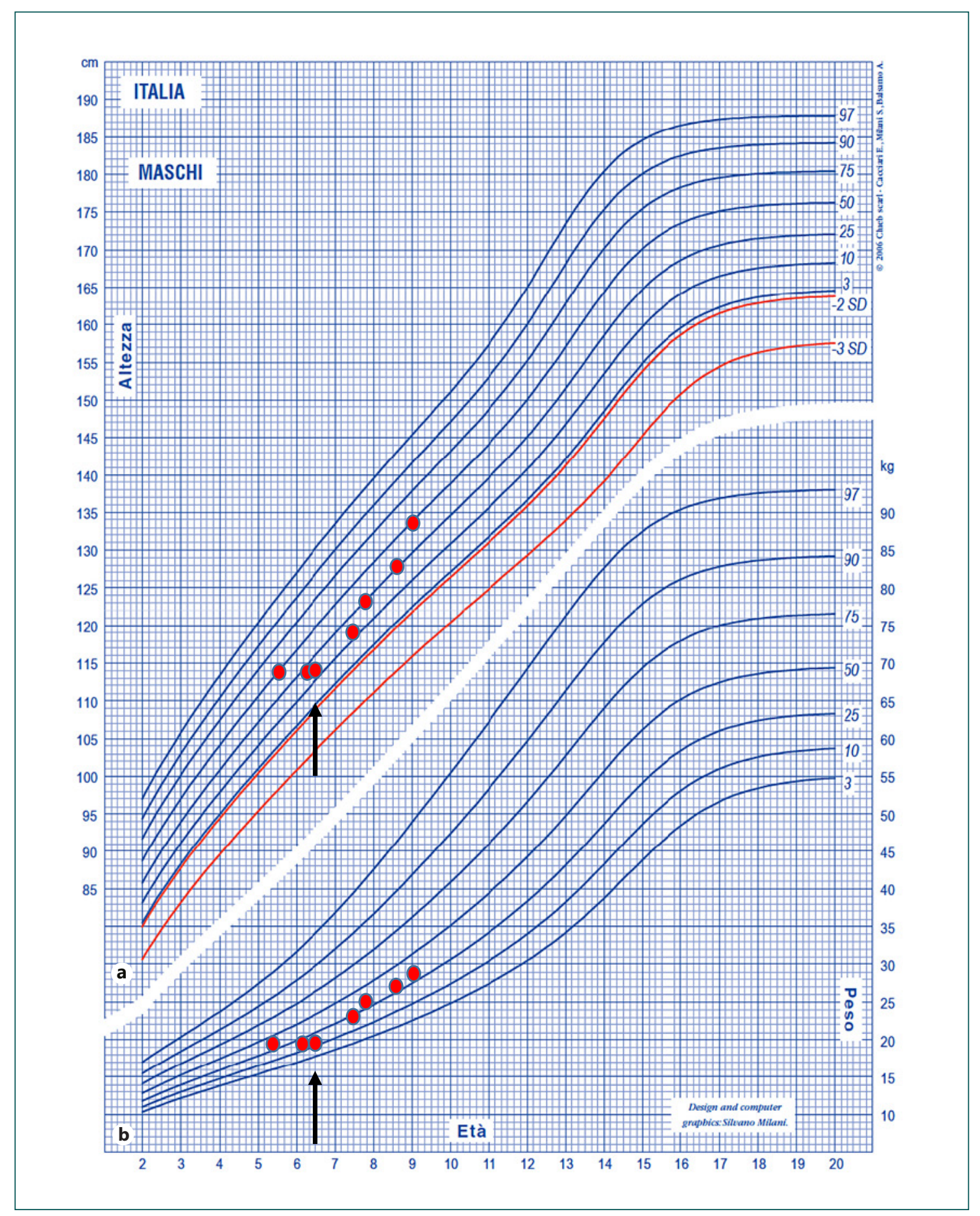

Abb. 1. (a) Körpergrößenperzentilen für Patient 1. (b) Gewichtsperzentilen für Patient 1. Die Pfeile in den Abbildungen zeigen an, wann die Kohlenhydratrestriktion beendet wurde.

Die Mutter gab gegenüber der Diätassistentin an, dass eine kohlenhydratarme Diät (40 g/Tag) begonnen worden war, um Insulininjektionen zu den Mahlzeiten während der Honeymoon-Phase zu vermeiden. Der HbAlc-Wert betrug $49 \mathrm{mmol} / \mathrm{mol}$, die TIR 66\% und die Zeit in Hypoglykämie 10\%; die tägliche Gesamtinsulindosis lag bei $0,34 \mathrm{U} / \mathrm{kg} /$ Tag. Die Ernährungsanalyse anhand eines 3-tägigen Ernährungsprotokolls ergab, dass die Gesamt- energieaufnahme bei etwa $60 \%$ des geschätzten Energiebedarfs von $1600 \mathrm{kcal}$ lag. Dabei stammten 17\% der Energie aus Kohlenhydraten, 30\% aus Protein und 53\% aus Fett.

Bei den folgenden Kontrollterminen berichtete die Mutter, dass sie mehr Kohlenhydrate in die Ernährung aufgenommen habe (200 g/Tag), allerdings besteht Grund zur Annahme, dass das Mädchen bis zum Alter von 8 Jahren, dem Ende der Honeymoon- 


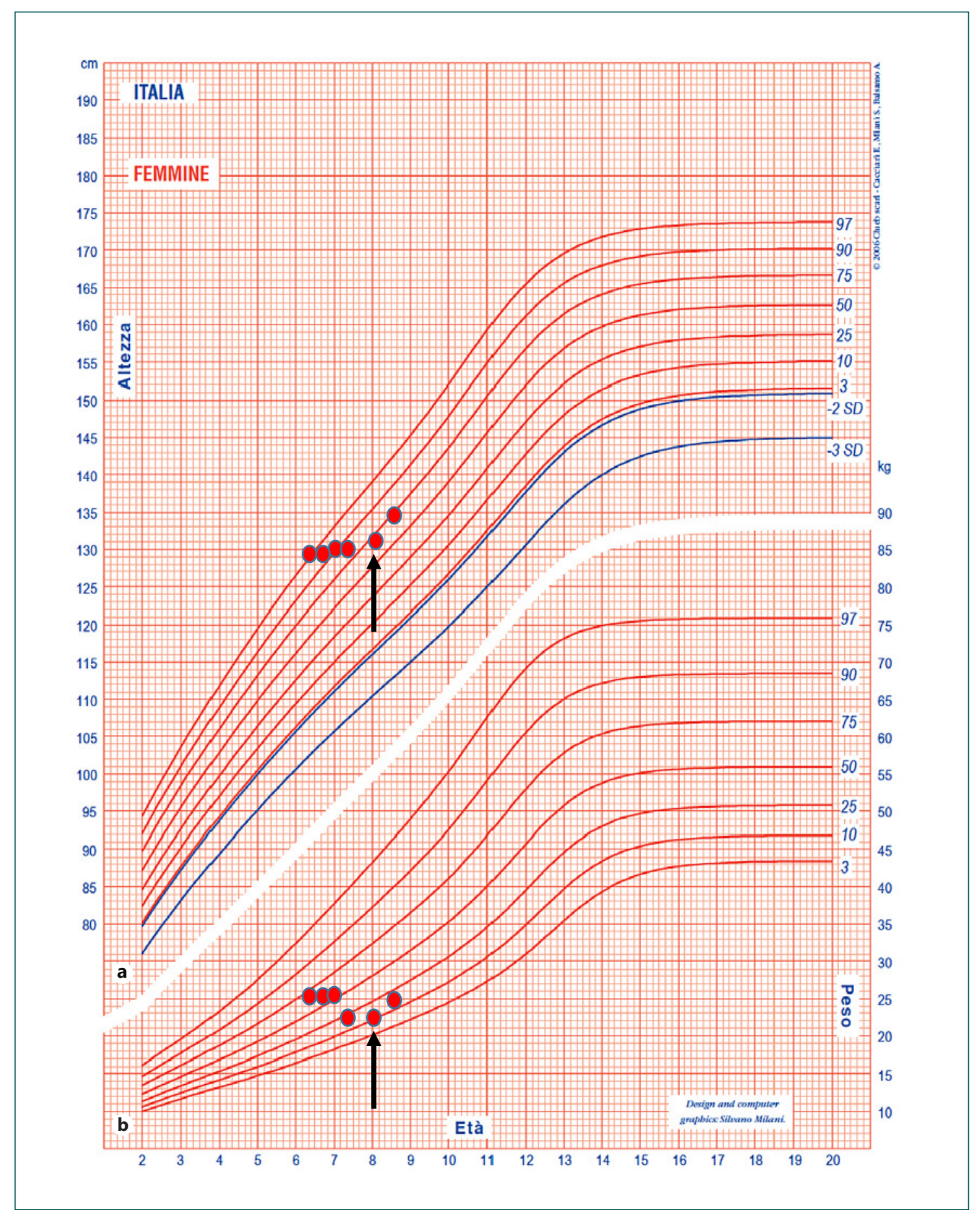

Abb. 2. (a) Körpergrößenperzentilen für Patient 2. (b) Gewichtsperzentilen für Patient 2. Die Pfeile in den Abbildungen zeigen an, wann die Kohlenhydratrestriktion beendet wurde.

Phase, nicht die adäquate Menge an Kohlenhydraten erhielt. Diese Patientin hatte für etwa 18 Monate eine kohlenhydratarme Kost erhalten. Ab dem Alter von 8 Jahren und 5 Monaten (nach einer Diabetes-Krankheitsdauer von 23 Monaten) setzte eine $\mathrm{Zu}$ nahme von Körpergewicht und -größe ein, wie in Abbildung 2 dargestellt; der HbAlc-Wert lag zwischen 47 und $50 \mathrm{mmol} / \mathrm{mol}$ und die Gesamtinsulindosis betrug 0,65 U/kg/Tag.

\section{Diskussion}

Diese beiden Fallberichte verdeutlichen die negativen Folgen einer Kohlenhydratrestriktion für das Längenwachstum. Die geringe Energieaufnahme wirkte sich bei diesen beiden Kindern mit Diabetes mellitus Typ 1 zuerst auf das Gewicht und einige Monate später auch auf die Körpergröße aus. Mit der Wiederaufnahme 
einer ausgewogenen Ernährung kehrte sich dieser Zustand ins Gegenteil um. Andere Ursachen für die Wachstumsverzögerung bei Typ-1-Diabetes, wie Hypo- und Hyperthyreose, Zöliakie, Nebenniereninsuffizienz und Wachstumshormonmangel, wurden ausgeschlossen.

In der Literatur findet sich nur eine von de Bock $\mathrm{M}$ et al. berichtete Fallserie [4]. Diese bestätigt die negativen Auswirkungen auf das Wachstum bei Kindern mit Typ-1-Diabetes und deutet darüber hinaus auf einen metabolischen Einfluss auf das Lipidprofil hin. Die Autoren berichteten über eine Serie von 6 Fällen, in denen die Umstellung auf eine kohlenhydratarme Ernährung zu Gewichtsverlust sowie zu erhöhten Nüchtern-Cholesterin- und Triglyceridwerten führte. Bei diesen Patienten machte die Kohlenhydrataufnahme etwa 6 bis 40\% der täglichen Energiezufuhr aus und die Ernährung deckte 70 bis $86 \%$ des geschätzten Energiebedarfs. Eine Kohlenhydratrestriktion führt wahrscheinlich zu einem Defizit bei der Energieaufnahme, das nicht durch andere Makronährstoffe (Fett und Protein) kompensiert wird und dies tritt bei Kindern eher auf als bei Erwachsenen, da Kinder wegen des Wachstums einen erhöhten Energiebedarf haben [4]. Darüber hinaus kann ein restriktives Essverhalten zu sozialer Isolation beitragen und $\mathrm{zu}$ einer weiteren Konfliktquelle in der Familie werden [4].

Demgegenüber hoben de Souza et al. [1] in einem kürzlich veröffentlichten Bericht hervor, dass die Einhaltung einer kohlenhydratarmen Ernährung ( $30 \mathrm{~g} / \mathrm{Tag}$ ) bei einem 5 Jahre alten Kind mit Typ-1-Diabetes mit einer optimalen glykämischen Kontrolle verbunden war und keine Nebenwirkungen hatte. Um eine ausreichende Nährstoffaufnahme sicherzustellen, wurde die Proteinmenge erhöht, bis das Kind nach den Mahlzeiten satt war: $70 \mathrm{~g}$ Protein, $30 \mathrm{~g}$ Kohlenhydrate und $55 \mathrm{~g}$ Fett pro Tag. Gemäß diesen Daten nahm der Junge täglich $900 \mathrm{kcal}$ auf, das heißt 60\% des geschätzten Energiebedarfs. Die durchschnittlichen Insulin-Tagesdosen wurden reduziert und den Autoren zufolge nahm er sehr gut an Körpergewicht und -größe zu [1].

Nach Angaben der internationalen Leitlinien sollten die Kohlenhydrataufnahme bei Kindern mindestens $45 \%$ der Energie ausmachen [2,3] und folgende Strategien eingehalten werden, um eine optimale postprandiale glykämische Kontrolle zu erreichen: entsprechend abgestimmte Insulin-Kohlenhydrat-Verhältnisse und Insulingaben, gesunde Nahrungsmittel als Kohlenhydrat- quellen wie Vollkornbrot und Vollkorngetreideprodukte sowie Hülsenfrüchte, Erhöhung der Ballaststoffaufnahme, Substitution von Kohlenhydraten mit niedrigem GI durch solche mit hohem GI und rechtzeitige präprandiale Insulinverabreichung 15 bis 20 Minuten vor einer Mahlzeit [5]. Die Leitlinien betonen, dass bei Kindern und Jugendlichen mit Typ-1-Diabetes keine zu starke Kohlenhydratrestriktion erfolgen sollte, da diese schädliche Auswirkungen auf das Wachstum, ein erhöhtes metabolisches kardiovaskuläres Risikoprofil sowie ein erhöhtes Risiko für gestörtes Essverhalten zur Folge haben kann [2, 3]. Wir möchten hervorheben, dass nach unseren Erfahrungen beim Management dieser beiden Fälle die Schulung der Familien von Kindern mit Typ1-Diabetes eine entscheidende Rolle spielt, besonders wenn sich die Kinder gerade in der Honeymoon-Phase befinden oder diese zu Ende geht. Eltern und Kinder könnten versucht sein, die Kohlenhydrataufnahme zu reduzieren, um Insulininjektionen zu vermeiden und/oder postprandiale Blutzuckerspitzen zu verringern, was allerdings eine verminderte Energieaufnahme zur Folge hat und zu Wachstumsstörungen führt.

\section{Ethikerklärung}

Die vorliegende Untersuchung am Menschen wurde in Übereinstimmung mit allen einschlägigen nationalen Vorschriften, institutionellen Richtlinien und gemäß den Grundsätzen der Deklaration von Helsinki durchgeführt. Für den vorliegenden Fallbericht ist keine Genehmigung durch die Ethikkommission unserer Einrichtung erforderlich; es wurde jedoch die schriftliche Einwilligung der Familien der Patienten eingeholt.

\section{Interessenkonflikte}

Es wurden keine Interessenkonflikte angegeben.

\section{Finanzierung der Studie}

Die Autoren haben keine Finanzierung für diese Arbeit erhalten.

\section{Autorenbeiträge}

Alle Autoren übernehmen Verantwortung für den gesamten Inhalt des eingereichten Manuskripts und haben die Einreichung freigegeben. R. F., C. R., V. C. und F. B. behandelten die Patienten; A. L. und M. S. erstellten das Manuskript.

\section{Literatur}

Die Literatur ist unter www.karger.com/doi/10.1159/000516251 abrufbar. 